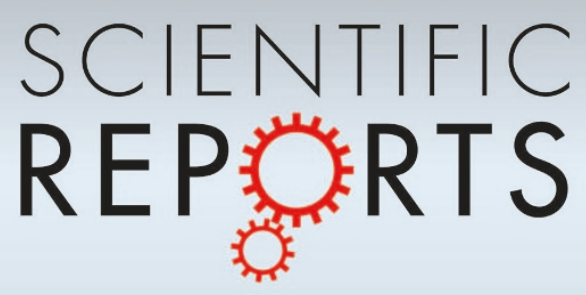

OPEN

SUBJECT AREAS:

MATERIALS SCIENCE

NANOSCALE MATERIALS

GRAPHENE

SYNTHESIS OF GRAPHENE

Received

14 June 2013

Accepted

24 July 2013

Published

3 September 2013

Correspondence and requests for materials should be addressed to E.-H.Y. lehyang@ieee. org)

\section{On the growth mode of two-lobed curvilinear graphene domains at atmospheric pressure}

\author{
Kitu Kumar \& Eui-Hyeok Yang
}

Department of Mechanical Engineering, Stevens Institute of Technology, Hoboken, New Jersey 07030.

We demonstrate the chemical vapor deposition (CVD) growth of 2-lobed symmetrical curvilinear graphene domains specifically on $\mathrm{Cu}\{100\}$ surface orientations at atmospheric pressure. We utilize electron backscattered diffraction, scanning electron microscopy and Raman spectroscopy to determine an as-yet unexplored growth mode producing such a shape and demonstrate how its growth and morphology are dependent on the underlying $\mathrm{Cu}$ crystal structure especially in the high $\mathrm{CH}_{4}: \mathrm{H}_{2}$ regime. We show that both monolayer and bilayer curvilinear domains are grown on $\mathrm{Cu}\{100\}$ surfaces; furthermore, we show that characteristic atmospheric pressure CVD hexagonal domains are grown on all other $\mathrm{Cu}$ facets with an isotropic growth rate which is more rapid than that on $\mathrm{Cu}\{100\}$. These findings indicate that the $\mathrm{Cu}$-graphene complex is predominant mechanistically at atmospheric pressure, which is an important step towards tailoring graphene properties via substrate engineering.

T he unique electronic and mechanical properties of graphene have made this 2-D material a test-bed for novel laboratory physics experiments as well as a contender for applications such as transparent electrodes ${ }^{1}$, supercapacitors ${ }^{2}$, high-speed electronics ${ }^{3}$, plasmonics ${ }^{4,5}$, photovoltaics ${ }^{6,7}$, photodetectors ${ }^{8,9}$, and large scale transistors ${ }^{10}$. The majority of physics research has been conducted on mechanically exfoliated graphene, but has limitations in practical applications due to the small areas achieved by this fabrication method. Therefore, efforts have focused on large-area growth techniques such as epitaxy on various substrates ${ }^{11,12}$ and metal-catalyzed thermal decomposition of hydrocarbons. Growth using this latter method, also known as CVD especially on copper $(\mathrm{Cu})^{13}$, has generated much interest due to the low cost of the $\mathrm{Cu}$ foil, ease of large-area growth at relatively low temperatures and ease of transfer to various substrates for device applications ${ }^{14,15}$. Studies of the fundamental CVD processes driving graphene growth such as system thermodynamics and growth kinetics including surface diffusion ${ }^{16}$, active species adsorption/desorption ${ }^{17}$ and edge-oriented crystal growth ${ }^{18}$, have been conducted to benefit graphene synthesis by reducing grain boundaries and defects such that CVD graphene quality can approach that of mechanically exfoliated graphene. Thus, the dynamics of the graphene/ $\mathrm{Cu}$ interface during growth are the subject of intensive examination. Of great interest are the shapes and alignment of graphene domains ${ }^{16,19,20-22}$ on $\mathrm{Cu}$ surfaces which directly influence the mechanical, chemical, and electrical properties of the coalesced polycrystalline graphene film ${ }^{23}$.

A recent work showed that monolayer graphene grown on single crystal $\mathrm{Cu}(111)$ under ultra high pressure chemical vapor deposition formed a continuous sheet with few grain boundaries, while that grown on single crystal $\mathrm{Cu}(100)$ was non-continuous and displayed exposed domain edges poorly aligned with the underlying $\mathrm{Cu}$ direction $^{24}$. The monolayer graphene grown under more typical low pressure (LPCVD) conditions usually has a 4-lobed, 4-fold-symmetric domain ${ }^{21,25}$, though large-area, dendritic growths have been demonstrated ${ }^{13}$. The 4-lobed domains growth mode was shown to be dominated by edge kinetics with a growth velocity dependent on the orientation of the graphene with respect to the underlying single crystal $\mathrm{Cu}(100)$ substrate $^{21}$. In addition, studies in atmospheric-pressure chemical vapor deposition (APCVD) graphene synthesis have demonstrated that the underlying crystal orientation in the typical polycrystalline Cu plays a greater role in domain growth kinetics and thus domain shape, size, and nucleation density than originally thought ${ }^{11,26}$. Typical domain shapes from literature in APCVD synthesized graphene are 6-fold symmetric hexagons for both monolayer ${ }^{27-29}$ and multilayer graphen ${ }^{27,30}$. However, regardless of $\mathrm{Cu}$ orientation, the growth mode of orientation-dependent domains remains largely unexplored.

Here we show the APCVD growth of 2-lobed curvilinear monolayer and bilayer domains exclusively with 1 -fold symmetry on $\mathrm{Cu}\{100\}$ surfaces. This finding reveals hitherto unexamined growth kinetics, which are 

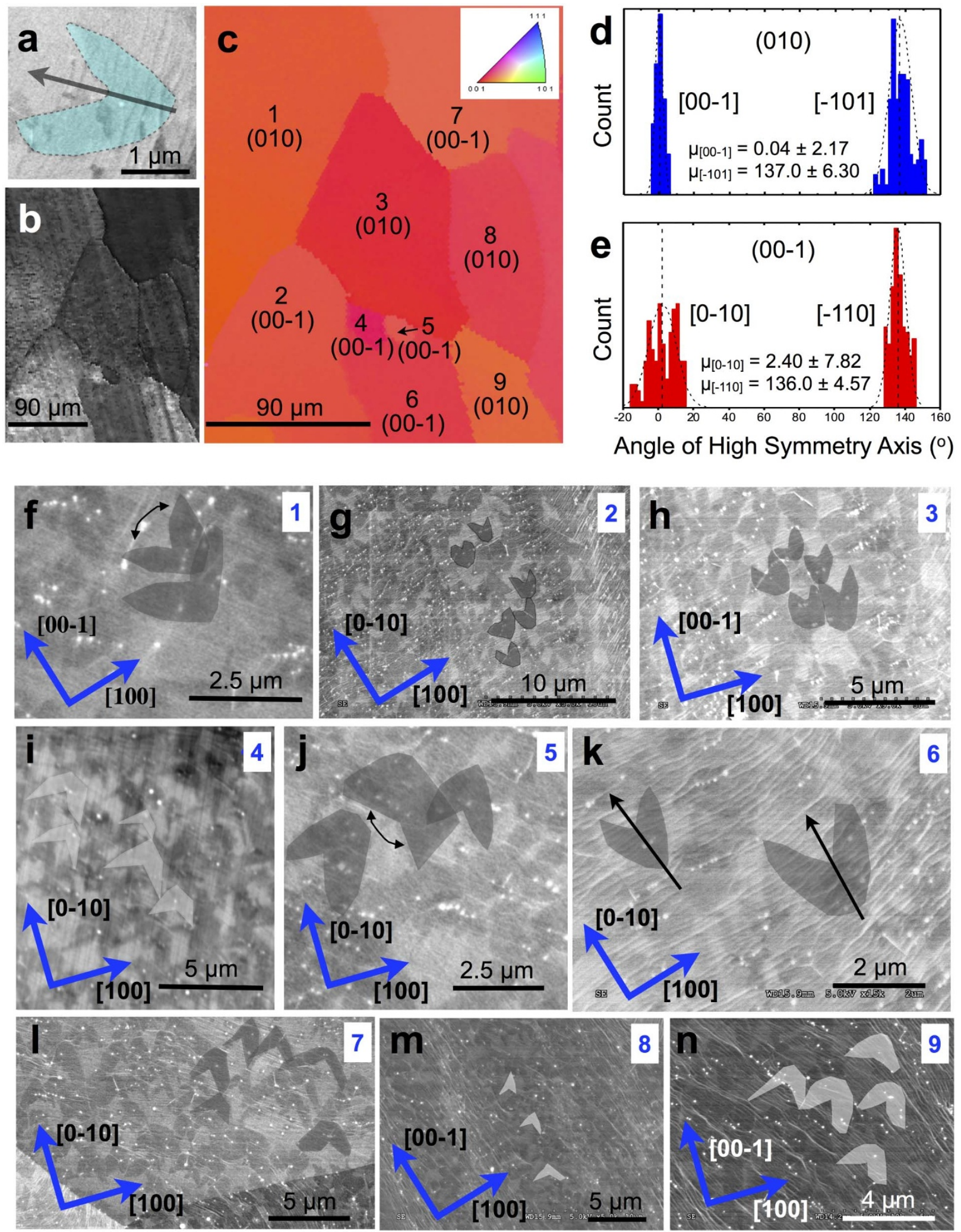

Figure 1 SEM and EBSD images of Cu grain effect on graphene domain shape. (a) Representative SEM image of a 2-lobed curvilinear monolayer domain grown under APCVD. (b) Low resolution SEM image of a high purity copper area with 2-lobed curvilinear domain growth. (c) Inverse pole EBSD map of the area in (b) indicating that the 2-lobed domains grow predominantly on the (010) and (00-1) surfaces. Inset to (c) is the color key used to determine surface orientation. (d) Alignment of the high-symmetry axis of domains on $\mathrm{Cu}(010)$ is along primarily along [00-1] and [-101] whereas in (e) the high-symmetry alignment on $\mathrm{Cu}(00-1)$ is primarily along $[-110]$ with a secondary alignment along $[0-10]$. The dashed line is a normal fit to the data, where $\mathrm{n}=100$ and the values presented as error are the standard deviations from the mean, $\mu$. (f-n) SEM images of false-color highlighted monolayer 2-lobed graphene domains corresponding to the growth on each $\mathrm{Cu}$ grain mapped in (c). 
modulated to a greater extent than previously reported by surface energy anisotropy of $\mathrm{Cu}$ at atmospheric pressure. To elucidate the growth mode of this domain shape with respect to the $\mathrm{Cu}$ surface, we use a combination of electron backscattered diffraction (EBSD), scanning electron microscopy (SEM) and Raman spectroscopy. We discuss the effects of diffusion limited growth which affects the symmetry of these domains and also examine the angular dependence of the graphene axis due to surface anisotropy which controls the curvilinear morphology to fully determine the effect of the $\mathrm{Cu}$ lattice on growth kinetics. We show that this anisotropic growth mode is not pronounced on higher index $\mathrm{Cu}$ surfaces, since hexagonal domains are found under the same APCVD conditions on surfaces which are not $\{100\}$. Furthermore, we experimentally show that the anisotropic growth rate of the curvilinear domain is slower than the isotropic growth rate of hexagonal/polygonal domains and attribute this to strain effects from lattice mismatch. Finally, we discuss how $\mathrm{Cu}$ surface energy may influence the bilayer growth mode, inducing similar growth kinetics, by straining of the intermediary monolayer graphene sheet.

\section{Results}

We investigated graphene growth under conditions chosen to elucidate the mechanism of anisotropic growth. Flow rates of $10 \mathrm{sccm}$ methane $\left(\mathrm{CH}_{4}\right)$ and $30-70 \mathrm{sccm} \mathrm{H}_{2}$ in $1000 \mathrm{sccm}$ Ar were maintained during the growth phase, which was terminated after $12 \mathrm{~s}$ or $25 \mathrm{~s}$ to examine individual domains before they coalesced into a continuous film. Figure 1a provides an SEM image of an isolated 2-lobed curvilinear structure after $25 \mathrm{~s}$ of growth. These distinctive morphologies were grown only on the $\mathrm{Cu}\{100\}$ surfaces (Figure 1c) of high purity $\mathrm{Cu}$ (99.999\% Alfa Aesar). The high symmetry axis of the domains was found to be well aligned, as shown in Figure 1d,e, along $[00-1]$ and $[-110]$ with secondary alignment along $[-101]$ and [0-10], respectively. The high-symmetry axis was also found to be well aligned to the perpendicular intersect of $\mathrm{Cu}$ surface steps (Figure $1 \mathrm{k}, \mathrm{n}$ ). On $\mathrm{Cu}\{100\}$, domain growth was found to remain unbroken across rolling striations, surface steps, and grain boundaries, though these surface features increased graphene nucleation density and played a role in morphology variation. Additionally, vicinity to other graphene domains had well-defined effects on single lobed morphology. The growth and presence of the curvilinear domain shape under APCVD conditions is puzzling; we elucidate the origin of this shape and growth mode below.

\section{Discussion}

LPCVD studies often produce lobed or flower-like graphene domains $s^{31}$ on $\mathrm{Cu}(100)$ orientations. In these cases, growth has been shown to be limited by angularly dependent edge kinetics, i.e., alignment of the fast growth direction of individual lobes with both the slow growth direction and $\mathrm{Cu}$ orientation produces 4-lobed morphologies, such that the flakes formed are not single crystals ${ }^{21}$. On the other hand, in APCVD growth, the hexagon/polygonal single crystal domain morphologies typically reported are limited by edge selectivity during growth, wherein the carbon attachment to only armchair edges is energetically stable, thus converting these edges to the zigzag orienta$\operatorname{tion}^{32}$. The six-fold symmetric growths reported in these studies is largely independent of $\mathrm{Cu}$ surface orientation, except in one case where the growth temperature was reduced, thereby growing 2-fold symmetric rectangular domains on only $\mathrm{Cu}(111)^{33}$. However, in that study, the growth mechanism was not clearly elucidated.

In our study, the distinct case of curvilinear growth specifically on the $\mathrm{Cu}\{100\}$ facets may be influenced by two causes. One is the ratio of $\mathrm{CH}_{4}: \mathrm{H}_{2}$ pressure which, in the case of the experiments presented here, is higher than typically found in literature. According to Vlassiouk et al. ${ }^{27}$, at a fixed $\mathrm{CH}_{4}$ partial pressure, greater $\mathrm{H}_{2}$ partial pressures would result in a distinct hexagonal shape of domains with zigzag edges since hydrogen etching of graphene is most efficient along this orientation ${ }^{27,34}$. In these studies, carbon concentrations are in the parts per million, whereas our experiments have orders of magnitude greater $\mathrm{CH}_{4}$ concentrations as described above. Therefore, we are operating in a different growth regime, and thus hydrogen etching can be ruled out as the predominant mechanism for the curvilinear shape. The second cause is more complex and involves several growth kinetic processes, indicating an unstudied growth mode on $\mathrm{Cu}(100)$ at atmospheric pressure. To examine this growth mode, we scrutinize the following phenomena:

1) Mobile carbon atoms at the $\mathrm{Cu}$ surface can diffuse along one of the four equivalent lattice directions of the substrate which would extend the domain growth along one of these directions ${ }^{35}$. Indeed, graphene has already been proven to align in two directions on $\mathrm{Cu}(100)$ under both LPCVD and APCVD $22,26,35,36$ though the angle between the two directions can be smeared anywhere from $30-90^{\circ}$ depending on the presence of step bunches or other surface interruptions. In our work, the two fast growth axes of the curvilinear domains (Figure 2a) are separated by a mean angle of $86.6^{\circ}$ relative to each other (Figure 2b). Coupled with the alignment of the domain high symmetry axes with either [00-1] or [-101] on $\mathrm{Cu}(010)$ and either $[-110]$ or $[0-10]$ on $\mathrm{Cu}(00-1)$ indicates that the two fast growth fronts are most efficient along the $<101>$ (Figure $2 \mathrm{c}$ ) or $<100>$ directions. This efficiency is attributed to surface energy anisotropy stemming from electron density correlations between the $\mathrm{Cu}$ lattice directions with highest atomic density and the graphene fast growth axes ${ }^{26}$.

2) If the domain growth is diffusion limited, i.e. directly influenced by surface energy anisotropy, the observed shape of a curvilinear lobe should be well fit to the function describing the angular dependence of the fast growth velocity to surface anisotropy ${ }^{21}$. Here, the velocity of the fast growth axis depends on the relative orientation of the graphene and $\mathrm{Cu}^{21}$. The model is described as

$$
v(\theta)=(1+r)+(1-r) \cos (\theta)
$$

where $v$ is the growth velocity, $\theta$ is the angle between the edge normal and slow growth direction, and $r$ is the ratio of the velocities in the slow and the fast directions and is the anisotropy factor for the 4 -fold symmetric $\mathrm{Cu}(100)$ surface. The purely fast axis mean velocity was determined to be $1.13 \pm$ 0.67 (s.d.) $\mu \mathrm{m} / \mathrm{s}$ from domain enlargement between $12 \mathrm{~s}$ and $25 \mathrm{~s}$ growths (Figure 2d,e respectively). Using the well-known anisotropy factor $r=0.25$, which predicts a sharp tipped lobe and is prevalent in our domains as seen in Figure 2a, we found a close fit with our curvilinear shape (Supplementary Figure S1). This model demonstrates that the distinctive shape of the graphene lobes on $\mathrm{Cu}\{100\}$ emerges from a growth velocity which is indeed angularly dependent on surface anisotropy. Therefore, in the regime of high $\mathrm{CH}_{4}: \mathrm{H}_{2}$ growth, growth is dominated by surface diffusion and graphene orientation dependent kinetics, in sharp contrast to the reported APCVD 6-fold growth via edge selectivity $^{32}$. We attribute the presence of the 1-fold symmetry with 2-lobes (rather than 4-lobes) to growth downhill the steps perpendicular to the [00-1] and [-110] directions Upward growth is prevented here due to the higher activation energy ${ }^{37}$ required for diffusion. We also note that as the fast growth front approaches adjacent domains (Figure 2e), the axis is not deflected; rather, the slow growth velocity increases in that direction. The cause of this effect is unclear, but accounts for the substantial variations from the mean lobe-to-lobe angle in $25 \mathrm{~s}$ growth compared to $12 \mathrm{~s}$ growth as in Figure $2 \mathrm{~b}$.

We now show that 6-fold symmetric graphene growth occurs on all other $\mathrm{Cu}$ surfaces measured. Figure 3 details $12 \mathrm{~s}$ growths on higher index $\mathrm{Cu}$ facets (Figure 3a,d). Here, the domains largely 

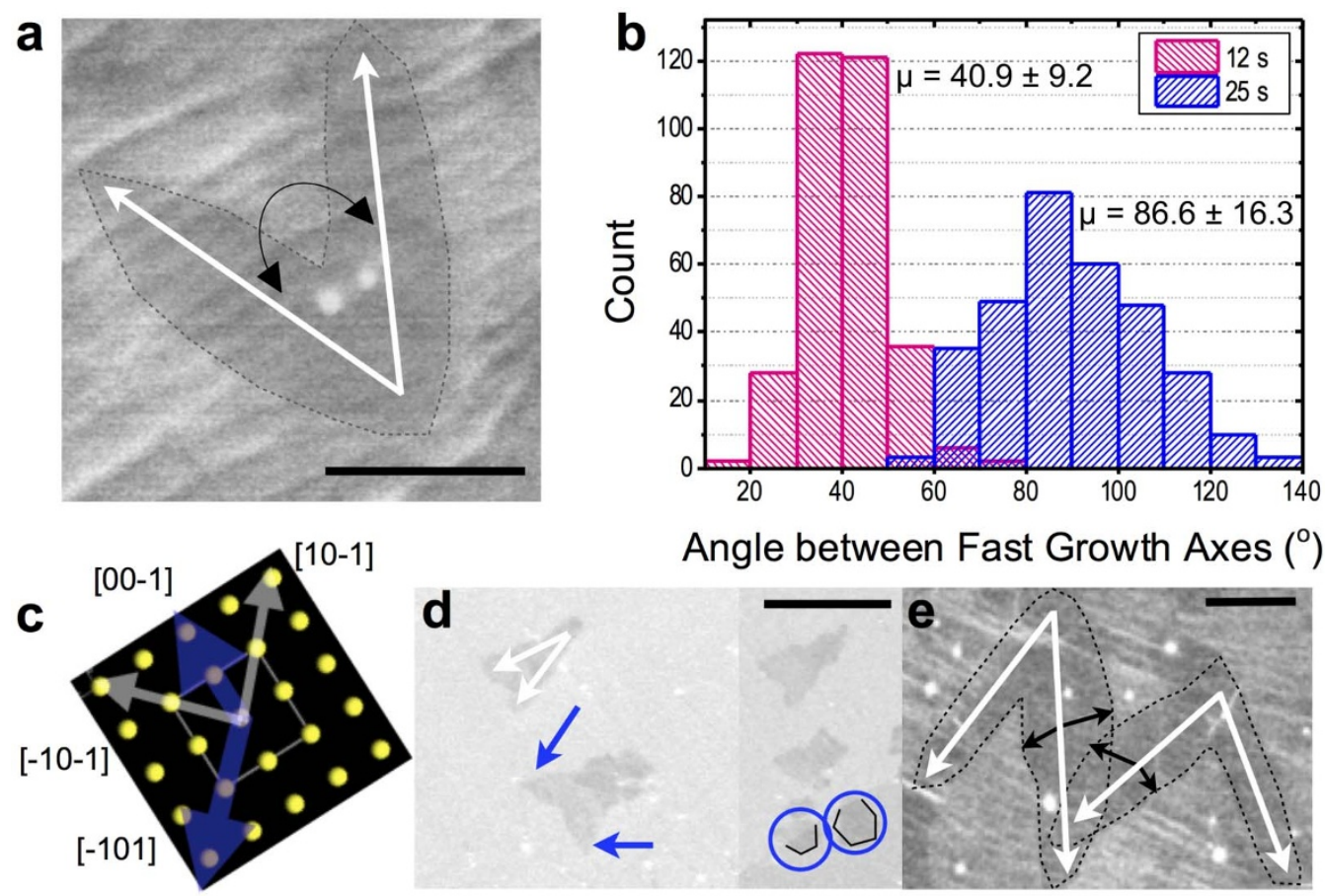

\section{Angle between Fast Growth Axes $\left({ }^{\circ}\right)$}

Figure 2 SEM images and data on 2-lobed curvilinear monolayer domain evolution. (a) A representative $25 \mathrm{~s}$ growth, 2-lobed curvilinear domain on $\mathrm{Cu}(010)$, with fast growth axes as indicated by white arrows. The curved black arrow represents the relative angle between the fast growth axes (lobes). (b) Histogram of angle between the fast growth axes of the curvilinear domains on $\mathrm{Cu}\{100\}$ surfaces after $12 \mathrm{~s}$ and $25 \mathrm{~s}$ of growth with mean values of $40.9^{\circ}$ and $86.6^{\circ}$, respectively. $\mathrm{n}=317$ and the values presented as error are the standard deviations from the mean, $\mu$. (c) Image of the (010) surface as in (a) with blue arrows denoting $\mathrm{Cu}$ directions, $[00-1]$ and $[-101]$, upon which the domains' high symmetry axes typically align. Grey arrows indicate direction of lobe growth, typically along $[-10-1]$ and [10-1], if the high symmetry axis is aligned to [00-1]. Evolution of domain morphology between (d) $12 \mathrm{~s}$ and (e) $25 \mathrm{~s}$ of growth. Fast growth axes marked with white arrows. (d) Appearance of branching in the precursor to the two lobed structure is indicated with blue arrows. Morphology change from curvilinear to hexagonal over a grain boundary is marked with blue circles. (e) Increase of slow growth rate as the fast growth front of one domain approaches another. Based on percentage of Cu covered by the graphene domain between $12 \mathrm{~s}$ and $25 \mathrm{~s}$ of growth, a growth velocity of $1.13 \pm 0.67 \mu \mathrm{m} / \mathrm{s}$ is calculated in the purely fast direction. Scale bars are (a) $1 \mu \mathrm{m}$, (d) $0.75 \mu \mathrm{m}$ and (e) $2.5 \mu \mathrm{m}$.

a

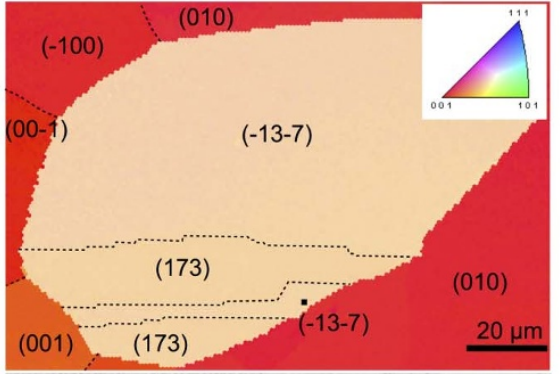

b

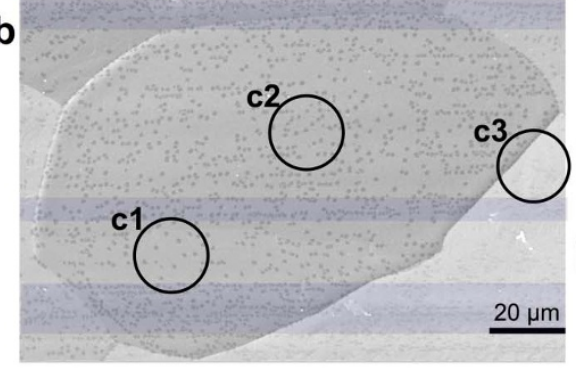

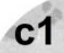

c1
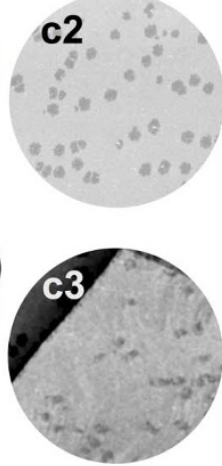

d

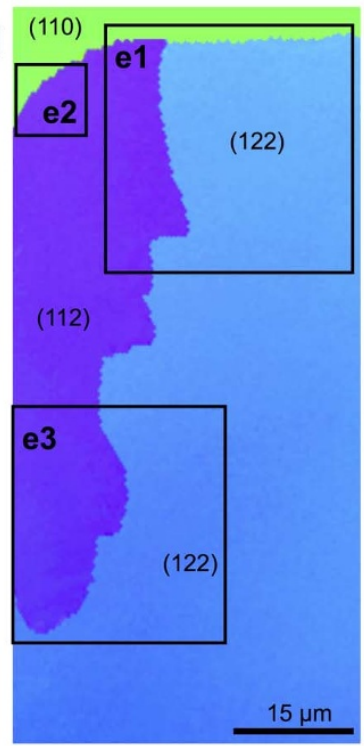

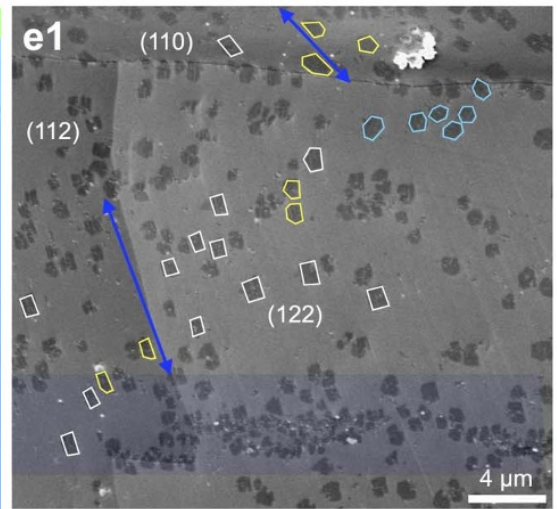

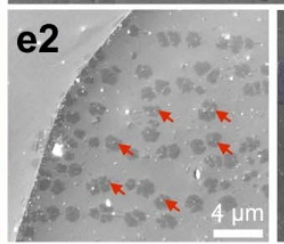

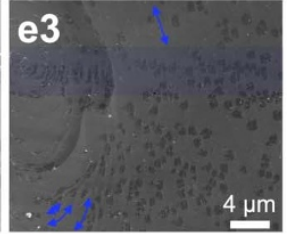

Figure 3 Graphene domain shape dependence on Cu orientation, 12 s growth. (a), (d) Inverse pole EBSD maps of predominantly non-(100) Cu facets. Inset to (a) is the color key used to determine surface orientation. (b), (c1-3), (e1-3) SEM images show that, under atmospheric pressure, hexagonal domains with predominantly isotropic growth trajectories are prevalent on high-index Cu facets such as (173), (122), and (112), with no evidence of the 2-lobed domains. (c1-3) SEM images of graphene domains whose low nucleation density is dependent on carbon concentration across the surface. (c3) Nucleation density of precursors to the 2-lobed curvilinear domain on (010) is lowest, confirming surface diffusion as the limiting factor in curvilinear growth. (e1-3) Details of high energy nucleation sites such as rolling striations (blue highlight) and nanoparticles (red arrows). (e1) Straining of growth trajectories due to surface steps (blue arrows) create morphological changes varying from predominantly isotropic growth domains (cyan hexagons) to moderately strained growth (yellow polygons) to 2-fold symmetric strained growth (white rectangles). 
nucleate on rolling-induced striations (and this density remains the same across the $\mathrm{Cu}$ facets) or steps and nanoparticles ${ }^{27}$, all energetically favorable points which reduce the activation energy for adsorption of carbon species ${ }^{38}$. However, in locations where these surface interruptions do not exist, (Figure 3c,1-2), nucleation density can be assumed to be directly proportional to carbon concentration on the surface. The low nucleation density on the high index $\mathrm{Cu}$ surfaces and even lower density on $\mathrm{Cu}(010)$, further confirms that growth is surface diffusion limited ${ }^{39}$. On higher index $\mathrm{Cu}$, the edges of the graphene domains appear to be randomly oriented. However, substantial morphology change occurs from hexagonal to rectangular shape (Figure $3 \mathrm{e}, 1$ ) as growth trajectories are strained ${ }^{39}$ parallel to the surface steps.

As mentioned above, electron density correlation between the graphene and $\mathrm{Cu}$ direction is fundamental to growth along specific axes. That is, $2 \mathrm{p} \mathrm{C}$ orbitals hybridize with the $3 \mathrm{~d} \mathrm{Cu}$ orbitals only in the directions which minimize lattice mismatch. Despite this growth efficiency, substantial strain is attributed to graphene grown on $\mathrm{Cu}(100)$ due to the sizeable lattice mismatch of a 6-fold symmetric crystal growing on a 4 -fold symmetric substrate $e^{40}$ and can be visualized through Moire patterns ${ }^{24,41}$. The presence of strain can be determined using Raman spectroscopy ${ }^{28,40}$. In Figure $4 c, d$, the intensity of the Raman map of the $G$ and $G$ ' full width-at-half-maximum (FWHM) is larger on the (100) surface than on (122) surface. Secondly, the Raman energy histograms (Figure 4e,f), of 12 s growth domains show substantial non-uniform strain on $\mathrm{Cu}(100)$ due to decrease of the Raman energy. Comparing this to the blue-shifts in the Raman bands of the hexagonal domains grown on $\mathrm{Cu}(122)$ clearly confirms the sizeable lattice mismatch of graphene on $\mathrm{Cu}(100)$, further supporting the case for the surface anisotropy modulated growth of 2-lobed curvilinear domains. Because the lattice mismatch minimization is more efficient on the higher energy $\mathrm{Cu}$ indices, it follows that graphene growth on these facets is more isotropic and faster than on the $\mathrm{Cu}(100)$. Indeed, our experiments corroborate this in Figure 4g; graphene coverage of the underlying $\mathrm{Cu}$ facet is shown to increase as the $\mathrm{Cu}$ index shifts away from the $\{100\}$ orientation.

Straining of the monolayer graphene lattice may lead to interesting kinetics when it comes to the growth of bilayers. Currently, the nature of the growth mechanism of bilayer graphene on monolayer under APCVD is not extensively studied ${ }^{42,43}$. Here, by extending the growth time from $25 \mathrm{~s}$ to 1 min under the high $\mathrm{CH}_{4}: \mathrm{H}_{2}$ regime, we observe the coalescence of the monolayer graphene domains to a continuous sheet and the growth of secondary domains atop this sheet. Interestingly, the 2-lobed curvilinear shape is present in these bilayer domains (Figure 5a,b) indicating similar kinetics of formation. The bilayer domains were not clearly visible under SEM, therefore the graphene sample was etch-released from the $\mathrm{Cu}$ substrate and transferred to $90 \mathrm{~nm} \mathrm{SiO}_{2}$ wafers for further analysis. We hypothesize that the growth process which occurs during monolayer growth i.e. surface diffusion and the graphene orientation- $\mathrm{Cu}$ direction dependent reactions are the same or similar during secondary layer growth. This strongly indicates that the $\mathrm{Cu}$ potential energy surface isotropy or anisotropy is transmitted to the intermediary a
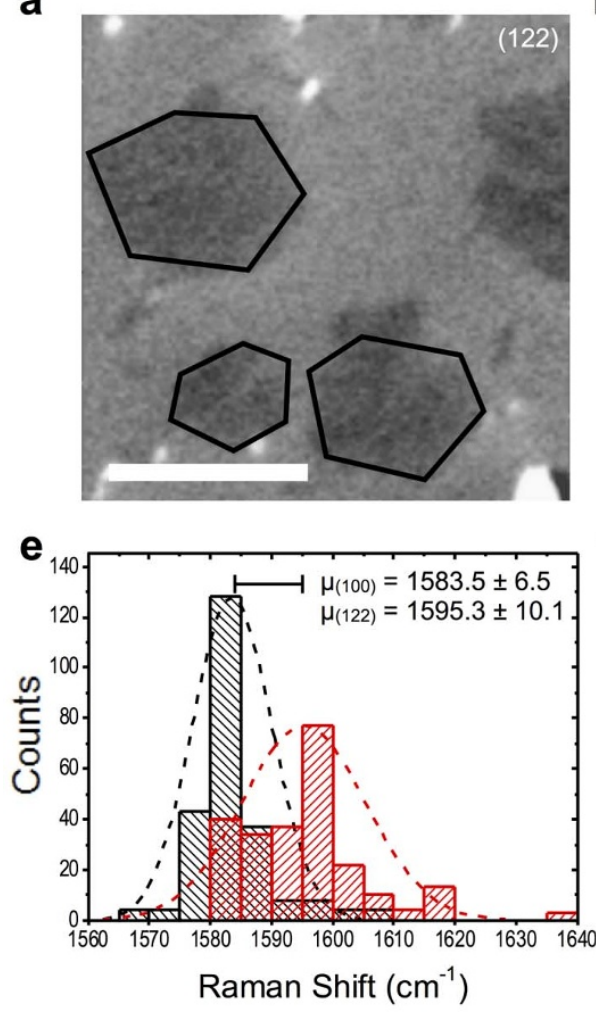

b

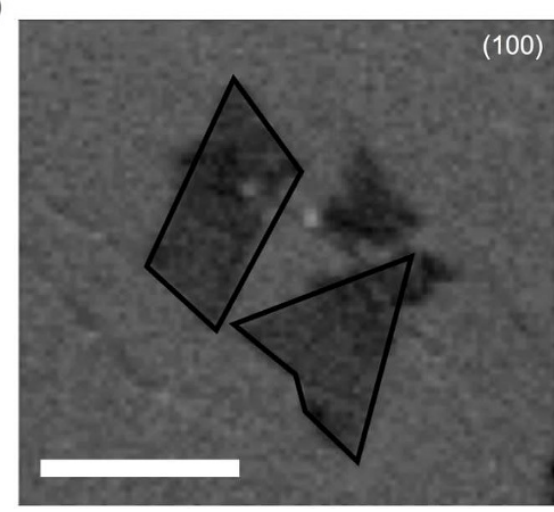

f

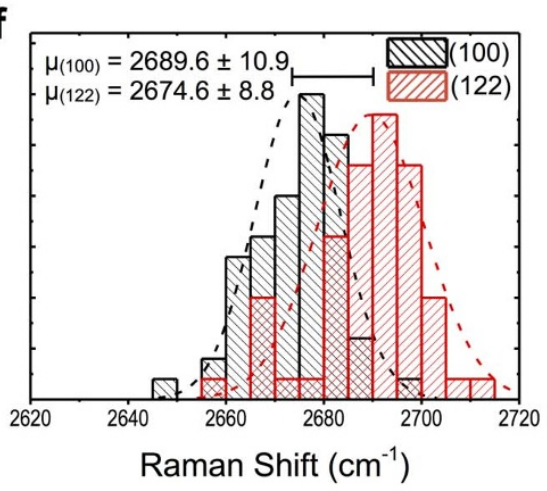

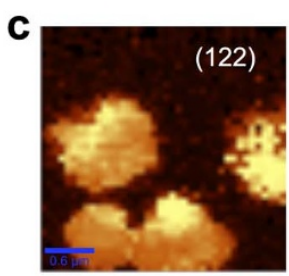
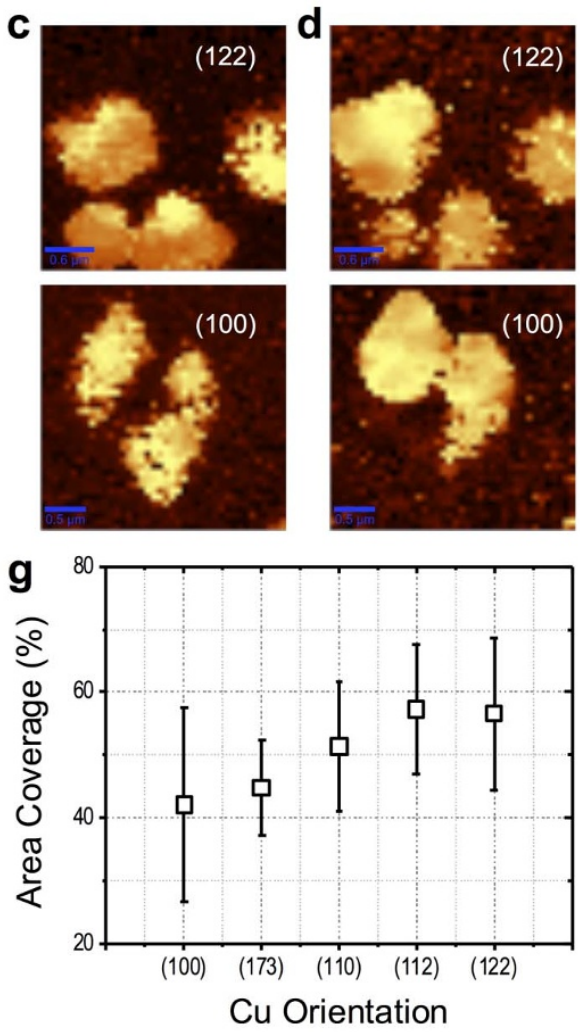

Figure 4 Strain and area coverage of graphene monolayer domains. (a), (b) SEM images of graphene domains on Cu(122) and (100), displaying

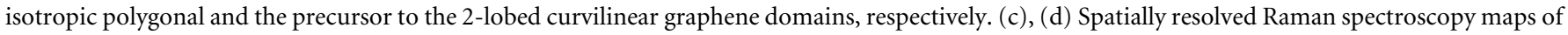

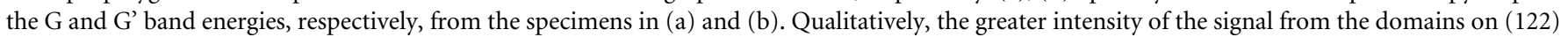

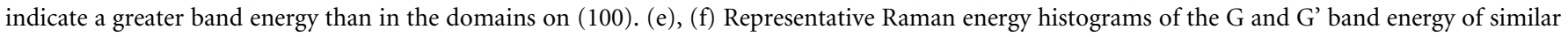

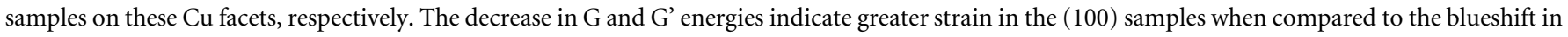

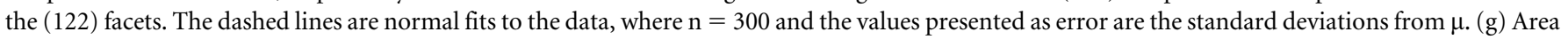

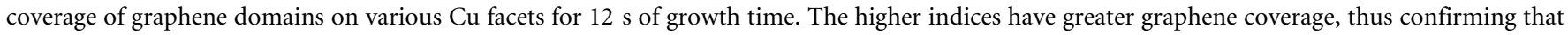

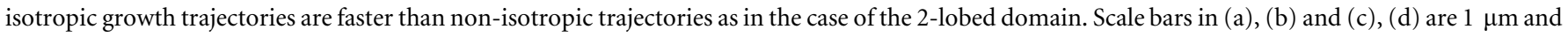
$0.5 \mu \mathrm{m}$, respectively. 

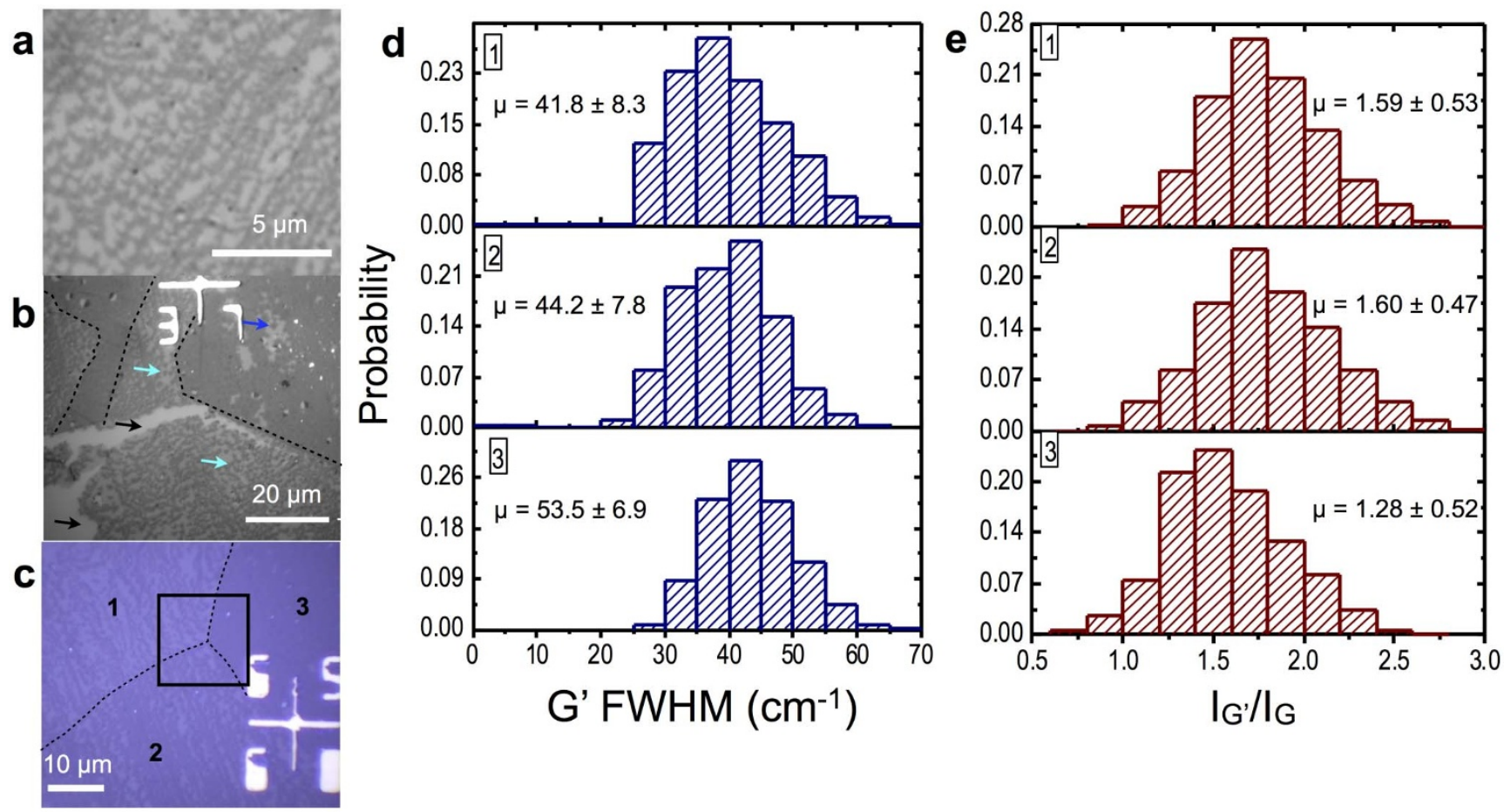

Figure 5 Evidence of Cu orientation influence on bilayer graphene growth. (a) Optical image of dense bilayer 2-lobed curvilinear graphene domains atop monolayer graphene transferred to $90 \mathrm{~nm} \mathrm{SiO}_{2}$ substrate. (b) Optical confirmation of multilayer growth. Black arrows indicate tear in graphene sheet and underlying $\mathrm{SiO}_{2}$. Cyan arrows indicate 2-lobed multilayer domains. Cu facet influence is seen on bilayer surface nucleation and diffusion kinetics, through the intermediary monolayer, as the areas marked by the dotted lines are regions of fast, isotropic growth. The blue arrow indicates a single hexagonal graphene domain. (c) Optical color image of transferred multilayer-atop-monolayer graphene with a triple point region of varying Cu facets, (100) in regions 1 and 2 and a high-index facet in region 3. Scale bar is $10 \mu \mathrm{m}$. (d,e) Histograms of G' FWHM and $\mathrm{I}_{\mathrm{G}} / \mathrm{I}_{\mathrm{G}}$ data extracted from the area marked by the black square in (c) indicating bilayer growth modes similar to monolayer graphene. Regions 1 and 2, which have lower bilayer domain nucleation density and coverage than the bilayer in region 3 (high index) also have lower $\mathrm{G}^{\prime} \mathrm{FWHM}$ and higher $\mathrm{I}_{\mathrm{G}^{\prime}} / \mathrm{I}_{\mathrm{G}}$ indicating less presence of bilayer material. As in the case of monolayer growth, the slower bilayer curvilinear domain anisotropic growth can be attributed to strain effects. $\mathrm{n}=500$ and the values presented as error are the standard deviations from the mean, $\mu$.

monolayer which influences approaching carbon species ${ }^{30}$. Though the effect of strain cannot be determined adequately since Raman spectra for polycrystalline graphene varies from single crystal graphene $^{44}$ and since the transfer process dopes the graphene $e^{45}$ which further affects the Raman bands, we note that $\mathrm{Cu}$ facet influence affects the nucleation density and growth rates of bilayer graphene in similar fashion to monolayer as evidenced in Figure 5b,c. The 2lobed bilayer domains grown on $\mathrm{Cu}\{100\}$ have lower nucleation density and smaller sizes than their hexagonal counterparts indicating slow, strained growth. Indeed, data extracted from the G' FWHM, G' and G intensity Raman maps (Supplementary Figure S2) of the region indicated in Figure 5c confirms greater bilayer coverage (Figure $5 \mathrm{~d}, \mathrm{e}$ ) in areas of fast isotropic growth by the greater incidence of broadened $\mathrm{G}^{\prime}$ band $\mathrm{FWHM}$ and lower $\mathrm{I}_{\mathrm{G}} / \mathrm{I}_{\mathrm{G}}$ intensity ratio. This result elucidates a similar strained growth mode of asgrown large area bilayer graphene on $\mathrm{Cu}\{100\}$ facets with high surface energy anisotropy, leading to interesting physics such as energy dispersion ${ }^{46}$ and bandgap tuning ${ }^{47}$ with applications in photonics devices such as photodetectors ${ }^{8,9}$ and photovoltaic cells ${ }^{6,7}$.

In summary, we have presented an analysis of a novel 2-dimensional graphene domain growth on $\mathrm{Cu}\{100\}$ facets whose unusual 2-lobed morphology and growth mode are controlled by the underlying surface orientation. We have shown that under the high $\mathrm{CH}_{4}: \mathrm{H}_{2}$ regime, the 2-lobed shape is largely controlled by surface diffusion and graphene orientation dependent kinetics, which are both influenced by the $\mathrm{Cu}(100)$ surface energy modulation along the high atomic density directions $<101>$ and $<100\rangle$. We have further shown that this growth mode rate is lower than the typical isotropic growth on higher index $\mathrm{Cu}$ facets, indicating the need for precise control of $\mathrm{Cu}$ orientation to grow high quality monolayer graphene. Finally, we have found that the growth of bilayer domains is similar to the monolayer growth mode and attribute this similarity to lattice straining of the intermediary graphene sheet. These findings indicate a hitherto unexplored anisotropic growth mode of graphene at atmospheric CVD conditions. Therefore, this work represents an important step in the fundamental understanding of the APCVD growth mechanisms for graphene as influenced by the underlying $\mathrm{Cu}$ orientation, which in turn will advance graphene synthesis for large-area applications.

\section{Methods}

The CVD graphene samples employed in this work were grown at atmospheric pressure on $25 \mu \mathrm{m}$ thick Cu foils (Alfa Aesar, 99.999\% purity) in a quartz tube furnace at $1000 \mathrm{oC}$ with $1000 \mathrm{sccm}$ of Argon, $30-70 \mathrm{sccm}$ of $\mathrm{H}_{2}$ and $10 \mathrm{sccm}$ of $\mathrm{CH}_{4}$ flowing during growth. To transfer graphene, 95,000 molecular weight PMMA (SigmaAldrich) dissolved to $4 \%$ in anisole was spin-coated on the graphene-Cu stack at a speed of $4000 \mathrm{rpm}$ for $1 \mathrm{~min}$ followed by $1000 \mathrm{rpm}$ for $1 \mathrm{~min}$ (acceleration of $1000 \mathrm{rpm} / \mathrm{s}$ ). The resulting PMMA thickness was approximately $50 \mathrm{~nm}$. The sample was then dried at $25^{\circ} \mathrm{C}$ in laboratory ambient air for $12 \mathrm{hrs}$ after which it was placed in a citric acid etchant bath (Transene, Inc) to remove the underlying $\mathrm{Cu}$. The PMMAgraphene stack placed in two successive water baths of $12 \mathrm{hrs}$ each and then transferred to a $90 \mathrm{~nm} \mathrm{SiO} 2 / \mathrm{Si}$ substrate.

1. Bae, S. et al. Roll-to-roll production of 30-inch graphene films for transparent electrodes. Nature Nanotech. 5, 574-578 (2010).

2. Kim, Y.-S., Kumar, K., Fisher, F. T. \& Yang, E.-H. Out-of-plane growth of CNTs on graphene for supercapacitor applications. Nanotechnology 23, 015301 (2011).

3. Liao, L. et al. High-speed graphene transistors with a self-aligned nanowire gate. Nature 467, 305-308 (2010). 
4. Wang, P. et al. Giant optical response from graphene-plasmonic system. ACS Nano 6, 6244-6249 (2012).

5. Echtermeyer, T. J. et al. Strong plasmonic enhancement of photovoltage in graphene. Nat. Commun. 2, 458 (2011).

6. Gomez De Arco, L. et al. Continuous, highly flexible, and transparent graphene films by chemical vapor deposition for organic photovoltaics. ACS Nano 4, 2865-2873 (2010).

7. Lee, Y.-Y. et al. Top laminated graphene electrode in a semitransparent polymer solar cell by simultaneous thermal annealing/releasing method. ACS Nano 5, 6564-6570 (2011).

8. Bao, Q. \& Loh, K. P. Graphene photonics, plasmonics, and broadband optoelectronic devices. ACS Nano 6, 3677-3694 (2012).

9. Withers, F., Bointon, T. H., Craciun, M. F. \& Russo, S. All-graphene photodetectors. ACS Nano 7, 5052-5057 (2013).

10. Petrone, N. et al. Chemical vapor deposition-derived graphene with electrical performance of exfoliated graphene. Nano Lett. 12, 2751-2756 (2012).

11. Sutter, P. W., Flege, J.-I. \& Sutter, E. A. Epitaxial graphene on ruthenium. Nature Mater. 7, 406-411 (2008).

12. Berger, C. Electronic confinement and coherence in patterned epitaxial graphene. Science 312, 1191-1196 (2006).

13. Li, X. et al. Large-area graphene single crystals grown by low-pressure chemical vapor deposition of methane on copper. J. Am. Chem. Soc. 133, 2816-2819 (2011).

14. Suk, J. W. et al. Transfer of CVD-grown monolayer graphene onto arbitrary substrates. ACS Nano 5, 6916-6924 (2011).

15. Li, X. et al. Transfer of large-area graphene films for high-performance transparent conductive electrodes. Nano Lett. 9, 4359-4363 (2009).

16. Bhaviripudi, S., Jia, X., Dresselhaus, M. S. \& Kong, J. Role of kinetic factors in chemical vapor deposition synthesis of uniform large area graphene using copper catalyst. Nano Lett. 10, 4128-4133 (2010).

17. Kim, H. et al. Activation energy paths for graphene nucleation and growth on $\mathrm{Cu}$. ACS Nano 6, 3614-3623 (2012).

18. $\mathrm{Wu}, \mathrm{B}$. et al. Equiangular hexagon-shape-controlled synthesis of graphene on copper surface. Adv. Mater. 23, 3522-3525 (2011).

19. Li, X. et al. Graphene films with large domain size by a two-step chemical vapor deposition process. Nano Lett. 10, 4328-4334 (2010).

20. Gao, L., Guest, J. R. \& Guisinger, N. P. Epitaxial graphene on $\mathrm{Cu}(111)$. Nano Lett 10, 3512-3516 (2010).

21. Wofford, J. M., Nie, S., McCarty, K. F., Bartelt, N. C. \& Dubon, O. D. Graphene islands on $\mathrm{Cu}$ foils: the interplay between shape, orientation, and defects. Nano Lett. 10, 4890-4896 (2010)

22. Nie, S., Wofford, J., Bartelt, N., Dubon, O. \& McCarty, K. Origin of the mosaicity in graphene grown on $\mathrm{Cu}(111)$. Phys. Rev. B 84, 155425 (2011).

23. Novoselov, K. et al. A roadmap for graphene. Nature 490, 192-200 (2012).

24. Zhao, L. et al. Influence of copper crystal surface on the CVD growth of large area monolayer graphene. Solid State Commun. 151, 509-513 (2011)

25. Li, X., Cai, W., Colombo, L. \& Ruoff, R. S. Evolution of graphene growth on Ni and Cu by carbon isotope labeling. Nano Lett. 9, 4268-4272 (2009).

26. Murdock, A. T. et al. Controlling the orientation, edge geometry, and thickness of chemical vapor deposition graphene. ACS Nano 7, 1351-1359 (2013).

27. Vlassiouk, I. et al. Role of hydrogen in chemical vapor deposition growth of large single-crystal graphene. ACS Nano 5, 6069-6076 (2011).

28. $\mathrm{Yu}, \mathrm{Q}$. et al. Control and characterization of individual grains and grain boundaries in graphene grown by chemical vapour deposition. Nature Mater. 10, 443-449 (2011).

29. Huang, P. Y. et al. Grains and grain boundaries in single-layer graphene atomic patchwork quilts. Nature 469, 389-392 (2011).

30. Robertson, A. W. \& Warner, J. H. Hexagonal single crystal domains of few-layer graphene on copper foils. Nano Lett. 11, 1182-1189 (2011).

31. Li, X. et al. Large-area synthesis of high-quality and uniform graphene films on copper foils. Science 324, 1312-1314 (2009).

32. Luo, Z., Kim, S., Kawamoto, N., Rappe, A. M. \& Johnson, A. T. C. Growth mechanism of hexagonal-shape graphene flakes with zigzag edges. ACS Nano 5, 9154-9160 (2011)

33. Wu, Y. A., Robertson, A. W., Schäffel, F., Speller, S. C. \& Warner, J. H. Aligned rectangular few-layer graphene domains on copper surfaces. Chem. Mater. 23, 4543-4547 (2011)
34. Zhang, Y., Li, Z., Kim, P., Zhang, L. \& Zhou, C. Anisotropic hydrogen etching of chemical vapor deposited graphene. ACS Nano 6, 126-132 (2012).

35. Rasool, H. I. et al. Atomic-scale characterization of graphene grown on copper (100) single crystals. J. Am. Chem. Soc. 133, 12536-12543 (2011).

36. Ogawa, Y. et al. Domain structure and boundary in single-layer graphene grown on $\mathrm{Cu}(111)$ and $\mathrm{Cu}(100)$ films. J. Phys. Chem. Lett. 3, 219-226 (2012).

37. Rasool, H. I. et al. Continuity of graphene on polycrystalline copper. Nano Lett. 11, 251-256 (2011)

38. Han, G. H. et al. Influence of copper morphology in forming nucleation seeds for graphene growth. Nano Lett. 11, 4144-4148 (2011).

39. Celebi, K. et al. Evolutionary kinetics of graphene formation on copper. Nano Lett. 13, 967-974 (2013).

40. He, R. et al. Large physisorption strain in chemical vapor deposition of graphene on copper substrates. Nano Lett. 12, 2408-2413 (2012).

41. Cho, J. et al. Atomic-scale investigation of graphene grown on $\mathrm{Cu}$ foil and the effects of thermal annealing. ACS Nano 5, 3607-3613 (2011).

42. Liu, L. et al. High-yield chemical vapor deposition growth of high-quality largearea AB-stacked bilayer graphene. ACS Nano 6, 8241-8249 (2012).

43. Liu, W., Li, H., Xu, C., Khatami, Y. \& Banerjee, K. Synthesis of high-quality monolayer and bilayer graphene on copper using chemical vapor deposition. Carbon 49, 4122-4130 (2011)

44. Bissett, M. A., Izumida, W., Saito, R. \& Ago, H. Effect of domain boundaries on the raman spectra of mechanically strained graphene. ACS Nano 6, 10229-10238 (2012).

45. Cheng, Z. et al. Toward intrinsic graphene surfaces: a systematic study on thermal annealing and wet-chemical treatment of $\mathrm{SiO}_{2}$-supported graphene devices. Nano Lett. 11, 767-771 (2011).

46. Wong, J.-H., Wu, B.-R. \& Lin, M.-F. Strain effect on the electronic properties of single layer and bilayer graphene. J. Phys. Chem. C 116, 8271-8277 (2012).

47. Choi, D. et al. Fully rollable transparent nanogenerators based on graphene electrodes. Adv. Mater. 22, 2187-2192 (2010).

\section{Acknowledgements}

The authors thank S. Strauf for use of his Raman spectroscopy and transmission measurement systems and J. Ding, A. Moy, and S. Brew for helpful comments. EBSD characterization was carried out at Lehigh University, Materials Science Department. Raman spectroscopy was carried out at the Center for Functional Nanomaterials, Brookhaven National Laboratory, which is supported by the U.S. Department of Energy, Office of Basic Energy Sciences, under Contract No. DE-AC02-98CH10886. This work was supported in part by the Robert Crooks Stanley Fellowship and National Science Foundation GK12 Fellowship at Stevens Institute of Technology. This work has also been supported in part by National Science Foundation (DMR-0922522, EECS-1040007, ECCS-1104870, and EEC-1138244) and Air Force Office for Scientific Research (FA9550-11-1-0272, FA9550-12-1-0326).

\section{Author contributions}

K.K. designed and carried out the experiments, performed data analysis and wrote the manuscript. K.K. and E.H.Y. discussed the results, reviewed the manuscript, and have given approval to the final version of the manuscript.

\section{Additional information}

Supplementary information accompanies this paper at http://www.nature.com/ scientificreports

Competing financial interests: The authors declare no competing financial interests.

How to cite this article: Kumar, K. \& Yang, E.-H. On the growth mode of two-lobed curvilinear graphene domains at atmospheric pressure. Sci. Rep. 3, 2571; DOI:10.1038/ srep02571 (2013).

This work is licensed under a Creative Commons Attribution 3.0 Unported license. To view a copy of this license, visit http://creativecommons.org/licenses/by/3.0 